\title{
A review on the role of gut microbiota in immune checkpoint blockade therapy for cancer
}

\author{
Esther $\mathrm{Kim}^{1} \cdot$ Hyeok Ahn ${ }^{2} \cdot$ Hansoo Park ${ }^{3}$ (i)
}

Received: 1 December 2020 / Accepted: 17 March 2021 / Published online: 30 March 2021

(c) The Author(s) 2021

\begin{abstract}
Gut microbiota has been studied in relation to human health and disease prediction for decades. Also, immune checkpoints (ICPs) are enthusiastically investigated for anti-tumor immunotherapy. Recent studies show potential of gut microbiome and gut cytokines as biomarkers for carcinogenesis and response prediction of immune checkpoint inhibitor (ICI) response. Evidence has revealed that intestinal microorganisms play a major role in the effectiveness of programmed cell death 1 (PD-1) and cytotoxic T lymphocyte-associated antigen 4 (CTLA-4) blockade. In this review, we have focused on how microbiome and microbiome-generated cytokines affect immune checkpoints. We have also described the molecular mechanisms behind this interplay and the bacterial strains that have a potential role in immunotherapy.
\end{abstract}

\section{Introduction}

Immune checkpoints (ICPs) provide signals that regulate antigen recognition by the $\mathrm{T}$ cells, thereby preventing the immune system from attacking cells uncontrollably ( $\mathrm{He}$ and Xu 2020). Located at the cell surface, ICPs alter the cell function upon binding the ligands secreted by other cells. Programmed cell death protein 1 (PD-1) and cytotoxic T lymphocyte-associated antigen 4 (CTLA-4) are co-inhibitory ICPs; they are expressed on activated tumor-specific $\mathrm{CD} 4^{+}$ cells and $\mathrm{CD} 8^{+} \mathrm{T}$-lymphocytes, and inhibit the progression of the immune reaction. In the absence of ICP proteins and their ligands, $\mathrm{T}$ cells function regularly to kill tumor cells. However, when tumor cells express the ligands for ICP proteins, they bind to the inhibitory proteins expressed on the $\mathrm{T}$ cells and inhibit the $\mathrm{T}$ cells from killing the tumor cells (Wei et al. 2018). Accordingly, the presence of an antagonist of the inhibitory signal known as the immune checkpoint inhibitor

Hansoo Park

hspark27@gist.ac.kr

1 California Institute of Technology, 1200 E California Blvd, Pasadena, CA 91125, USA

2 School of Life Science, Gwangju Institute of Science and Technology (GIST), Cheomdangwagi-ro 123, Buk-gu, Gwangju 61005, Korea

3 Department of Biomedical Science and Engineering, Gwangju Institute of Science and Technology (GIST), Cheomdangwagi-ro 123, Buk-gu, Gwangju 61005, Korea
(ICI) (a corresponding antibody that inhibits the receptor function) enhances the antitumor immunity. The most commonly used inhibitors for CTLA-4 include ipilimumab and tremelimumab, while those for PD-1 include pembrolizumab and nivolumab; the inhibitors for programmed death-ligand 1 (PD-L1), the primary ligand of PD-1, include atezolimumab, avelumab, and durvalumab. The ICPs are, therefore, exploited by the tumor cells as an immune escape mechanism, suppressing the adaptive immune responses. Stimulated expression of the antigens expressed in the tumor microenvironment result in an increased expression of ICP proteins, which in turn decreases the effectiveness of the antitumor immune responses by debilitating the cytotoxic and cytokine-producing ability of the T cells (Darvin et al. 2018). Thus, PD- 1 and CTLA-4 are attractive targets for anti-cancer immunotherapy.

While various biomarkers are known to reflect the tumor immune microenvironment, such as tumor mutational burden, mismatch-repair deficiency, and density of tumor-infiltrating lymphocyte (TIL), recently, both cytokines and gut microbiome have been extensively studied owing to their potential to serve as biomarkers for both cancer progression and response prediction to ICIs (Yi et al. 2018). Cytokines, released in response to infection and inflammation, play a critical role in modulating the development and progression of malignant cells by their complex and vast network. Interferon (IFN), the first discovered member of the cytokine family, which presently forms an essential part of the innate immune system, evokes anti-viral defense response against 
viral infections. Interleukins (ILs), another important family of cytokines, are responsible for various functions of the immune cells, including activation, proliferation, motility, and differentiation. Hence, they exhibit both pro- and antiinflammatory properties (Zhang and An 2007). While the immunological functions of most ILs are known, many are yet to be unveiled. A few ILs have biphasic effects and some even have differences in their function depending upon the type of the tumor cell present. In practice, cytokines have been implemented as biomarkers to observe cancer cell progression in various cancer patients.

Gut microbiota has a vital role in modulating the immune system responses. Furthermore, it has been reported that the gut microbiome essentially governs the efficacy of PD-L1. In this review article, various studies revealing the effect of the microbiome on the efficacy of PD- 1 and CTLA- 4 blockade have been reviewed. Negative impact of dysbiosis due to antibiotics on the efficacy of PD-1 and CTLA-4 blockade was also affirmed. Fecal microbiome transplant (FMT) from patients responsive to the ICI treatment resulted in improved efficacy of ICP blockade. These studies identified several bacterial genera imparting benefits to ICI treatment. Immune mechanisms that altered the efficacy of the ICP blockade treatment involved cytokines. The related studies have been summarized in Table 1.

\section{Main text}

Although immunotherapy is a long-used practice, it has recently achieved exceptional success against distinct hematological and solid metastatic malignancies (Waldmann 2003). It has been reported that the ICIs disrupt the interaction between the ligands and the $\mathrm{T}$ cell inhibitory receptors, inducing $\mathrm{T}$ lymphocyte-mediated immune responses (Pardoll 2015). PD-1 blockade with its corresponding monoclonal antibodies (mAbs) is remarkably effective against advanced melanoma, non-small cell lung cancer (NSCLC), and renal cell carcinoma (RCC) (Routy et al. 2018).

\section{PD-1 antibody immunotherapy and the effect of FMT on PD-1 antibody efficacy}

The study by Routy et al. ushered in a new perspective by connecting the field of microbiome and immunotherapy targeting PD-L1 (Routy et al. 2018). The prospect that primary resistance to PD-1 blockade may be affected by the microbial imbalance associated with antibiotic or malignant disease in tumor-bearing mice and cancer patients was investigated. The authors concluded that the aberrant composition of the gut microbiome can result in primary resistance to ICIs. The impact of antibiotics (ATBs) on cancer patients, specifically those having advanced NSCLC, RCC, or urothelial carcinoma, after they received PD-1/PD-L1 mAb treatments was studied. In the absence of ATBs, the tumor growth was significantly reduced when injected with PD-1 $\mathrm{mAbs}$. However, $\mathrm{mAbs}$ were essentially ineffective when the mice (with RET melanoma and MCA-205 sarcoma) were previously treated with ATB. Hence, antibiotic consumption was correlated to negative response to PD-1 blockade. Based on the response evaluation criteria in solid tumors (RECIST) version 1.1 (Eisenhauer et al. 2009), patients who received PD-1/PD-L1 treatment were segregated into two groups: responders (R) and non-responders (NR). FMT from the stool samples of these patients to ATB-treated mice revealed that the stool samples from the R patients induced sensitivity, while those from NR patients induced resistance to PD-1 blockade, which is commensurate with efficiency in ATB-treated or germ-free mice. Two weeks after the inoculation with fecal samples and MCA-205 tumor cells, the ATB-treated mice were subjected to treatment with PD-1 $\mathrm{mAbs}$. The mice that received FMT from $\mathrm{R}$ patients showed slower tumor growth than those that received FMT from NR patients. Between the two groups, the $\mathrm{R}$ group was enriched with several key bacterial genera and strains compared to the other. Akkermansia muciniphila, the most prominent commensal bacterium associated with positive clinical outcome, was found in both NSCLC and RCC patients. Enterococcus hirae 13144 (E. hirae) was also observed repeatedly in R-NSCLC patients. A combination of A. muciniphila and E. hirae led to the accumulation of central memory $\mathrm{CD}^{+}$ $\mathrm{T}$ cells expressing chemokine receptors CCR9 (associated with small intestine) and CXCR3 (associated with $\mathrm{T}$ helper 1 (Th1) immune response). Increased recruitment of these $\mathrm{CD} 4^{+} \mathrm{T}$ cells is dependent on IL-12 secretion induced by $A$. muciniphila and E. hirae.

\section{CTLA-4 blockade immunotherapy}

A similar study by Vetizou et al. revealed an IL-12-dependent Th1 immune response induced by Bacteroides fragilis, which resulted in an increased response to CTLA-4 blockade (Vétizou et al. 2015). The authors found that the CTLA-4 blockade-induced immune-stimulatory effects were dependent on the microbiome, which in turn was regulated by the mobilization of intestinal lamina propria $\mathrm{CD} 11 \mathrm{~b}^{+}$dendritic cells (DC) and IL-12-associated Th1 immune response. Germ-free mice and mice treated with a combination of antibiotics, including ampicillin, colistin, and streptomycin (ACS), were re-colonized with bacterial species associated with CTLA-4 antibody-treated intestinal mucosa. In both groups of mice, reduced activation of the $\mathrm{CD} 4^{+} \mathrm{T}$ cells and TILs in Ret melanoma and the MC38 colon cancer models indicated significantly reduced efficacy of CTLA-4 antibody. The $\mathrm{CD} 4^{+} \mathrm{T}$ cells that were harvested from the spleens of CTLA-4 antibody-treated mice, or from the blood of the 


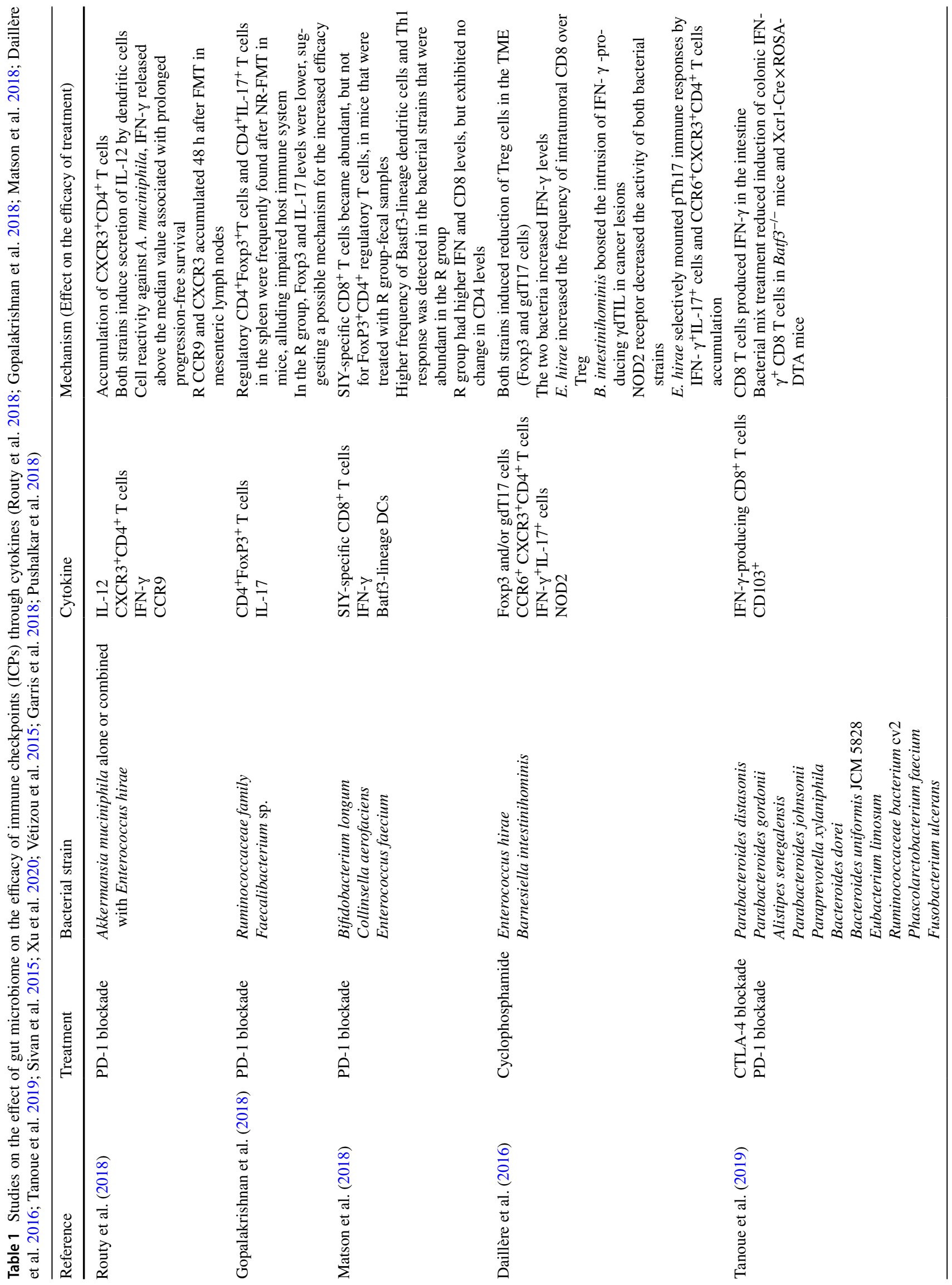




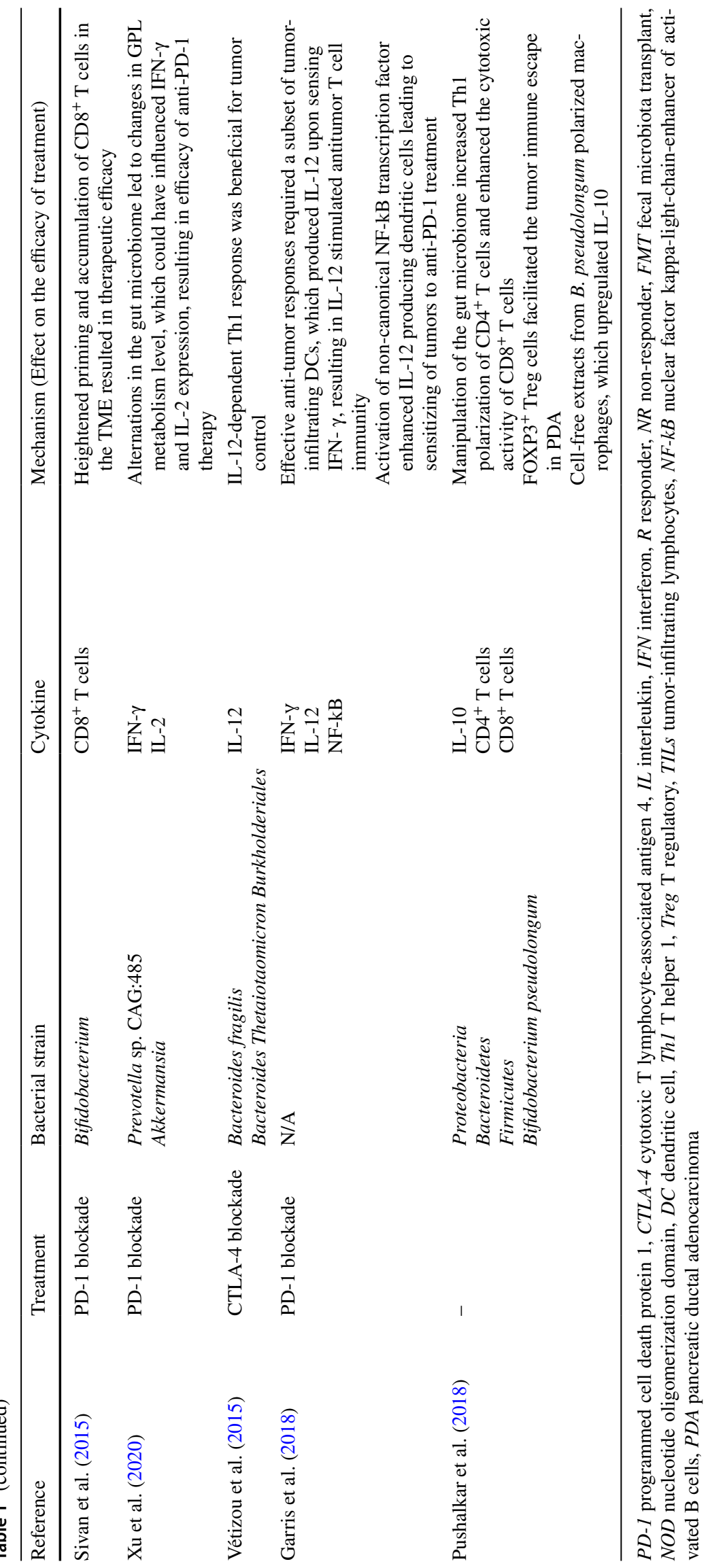


patients with metastatic melanoma (MM) or NSCLC after treatment with ipilimumab, demonstrated recovery of the Th1 phenotype. B. fragilis was observed to partially restore the efficacy of CTLA-4 antibody that was reduced due to the lack of gut microbiota. Abundance of $B$. fragilis was associated with reduced size of the tumor in the CTLA-4 blockade-treated mice. Hence, the microbiota composition influenced the efficacy of CTLA-4 blockade (B. fragilis and/ or B. thetaiotaomicron and Burkholderiales).

\section{The effect of specific bacterial strains, cytokines and immune cells on immunomodulation}

Garris et al. in their study reinforced the significant role of IL-12 in antitumor responses (Garris et al. 2018). The study findings revealed that the activation of antitumor $\mathrm{T}$ cells by anti-PD-1 is indirect, as it requires a crosstalk between the T cells and DCs. The crosstalk is, however, dependent on IFN gamma (IFN- $y$ ) and IL-12. Anti-PD-1 mAbs antagonize the PD-1 inhibitory pathway in T cells and, thereby, heighten the activation of $\mathrm{CD}^{+} \mathrm{T}$ cells. In turn, the DCs sense the IFN- $y$ released from the $\mathrm{mAb}$-activated $\mathrm{T}$ cells, instead of binding directly to the drug. DCs produce IL-12, which then stimulates the antitumor $\mathrm{T}$ cell immunity and sensitizes the tumors to anti-PD-1 treatment. Moreover, activation of the non-canonical nuclear factor kappa-light-chain-enhancer of activated B cells (NF-kB) transcription factor pathway augments the DCs that produce IL-12. Thus, NF-kB was identified as a potential therapeutic target for enhancing the checkpoint blockade response.

$\mathrm{Xu}$ et al. established the gut microbial genome and various plasma metabolites associated with PD-1 antibody immunotherapy in CT26 tumor-bearing mice. The $16 \mathrm{~S}$ ribosomal RNA (rRNA) gene sequencing, metagenomic shotgun sequencing, and non-targeted metabolomic analyses revealed that antibiotic treatment altered the taxonomic composition of the gut (Xu et al. 2020). An abnormal gut microbiome lowered the efficacy of PD-1 antibody immunotherapy. The relative therapeutic efficacy of PD-1 antibody treatment in combination with different antibiotics varied among the experimental groups: control group (good-response), vancomycin-treated (Vanc) group (medium-response), colistintreated (Coli) group (poor-response), and ACS-treated group mice (no-response). Prevotella sp. CAG: 485 and A. muciniphila were related to positive therapeutic immunotherapy efficacy. On the contrary, Bacteroides sp. and Bacteroides sp. CAG: 927 were associated with negative therapeutic efficacy. Similar to the observations by Routy et al. (2018), this study also revealed enhanced immunotherapeutic efficiency on enriching the intestine with Akkermansia sp. The better efficacy of PD-1 antibody was linked to the glycerophospholipid metabolic pathway, as changes in the gut microbiome altered the level of glycerophospholipid metabolism. Xu and his colleagues detected that while there was no change in the number of $\mathrm{CD}^{+}{ }^{+}$and $\mathrm{CD} 8^{+} \mathrm{T}$ cells, the cell IFN- $\gamma$ and IL-2 concentrations in the antibiotic-treated groups were significantly reduced compared to that in the control group. Thus, it was suggested that metabolism may affect the expression of immune-related cytokines, IFN-y and IL-2, in the tumor microenvironment, which then alters the therapeutic effect of PD-1 antibody. In addition, the metagenomic pathway function prediction demonstrated IL-17 secretion to be linked with negative immunotherapeutic efficacy.

Sivan et al. compared the effects of anti-PD-1/PD-L1 mAbs by observing the subcutaneous B16.SIY melanoma growth in genetically similar C57BL/6 mice obtained from Jackson Laboratory (JAX) and Taconic Farms (TAC) (Sivan et al. 2015), which harbored a disparate commensal microbiota. Aggressive tumor growth observed in TAC mice resulted from an immune-mediated response. Both the accumulation of intratumoral $\mathrm{CD} 8^{+} \mathrm{T}$ cells and the responses of tumor-specific $\mathrm{T}$ cells were lower in TAC mice than that in JAX mice. Transfer of JAX fecal material into TAC mice alone was sufficient to reduce the tumor growth rate and increase the infiltration of the $\mathrm{CD} 8^{+} \mathrm{T}$ cells. When the JAX fecal material was combined with PD-L1, tumor control and tumor antigen-specific $\mathrm{T}$ cell responses were enhanced. This indicates that the composition of the commensal microbiome affects the antitumor immunity and the efficacy of anti-PD-L1 mAb. The only genus-level taxon that significantly correlated with the accumulation of activated antigen-specific $\mathrm{T}$ cells was Bifidobacterium. The strongest association was reported with antitumor $\mathrm{T}$ cell response, and it significantly increased with the transplant of JAX fecal material into TAC. The positive effect in JAX mice could be attributed to Bifidobacterium. When the mice were treated with Bifidobacterium, tumor control was notably enhanced. The mechanism of this therapeutic effect is rather indirect, mediated by the host antitumor T cell responses, as Bifidobacterium treatment led to extensive induction of tumorspecific T cells. However, Bifidobacterium had no effect in CD8-depleted mice. TAC mice inoculated with B16 parental tumor cells or MB49 bladder cancer cells, when subjected to treatment with Bifidobacterium displayed delayed tumor growth. Hence, administration of a cocktail of Bifidobacterium sp. in TAC mice containing melanoma was identified as a positive promoter of antitumor response. Further, in combination with ICI, tumor growth significantly decreased. In both JAX and Bifidobacterium-treated TAC mice, an augmented proportion of major histocompatibility complex (MHC) Class II ${ }^{\text {hi }}$ DCs and IFN- $y$ were reported in the tumors.

Matson et al. examined baseline stool samples from metastatic melanoma patients to evaluate the association between commensal bacterial composition and clinical efficiency of PD-1 blockade immunotherapy (Matson et al. 2018). Similar 
to the study by Routy et al. (2018), the patients were categorized into R and NR groups based on the RECIST 1.1 version. Three weeks after inoculation with B16.SIY melanoma cells, the $\mathrm{R}$ microbiota-reconstituted mice exhibited a significant increase in SIY-specific $\mathrm{CD} 8^{+} \mathrm{T}$ cells (thereby augmenting priming of tumor antigen-specific $\mathrm{CD} 8^{+} \mathrm{T}$ cells), but no change was observed in $\mathrm{FoxP}^{+} \mathrm{CD}^{+}$regulatory $\mathrm{T}$ cells. IFN-y enzyme-linked immunosorbent spot of ex vivo SIY-stimulated splenocytes also exhibited an increased frequency of activated T cells. While anti-PD-L1 efficacy was enhanced in mice colonized with $\mathrm{R}$ microbiota, mice colonized with NR microbiota exhibited completely ineffective anti-PD-L1. Slower baseline tumor growth in two of the three R-derived mouse cohorts was congruent with the findings of the study by Sivan et al. (2015) that reported FMT from $\mathrm{R}$ alone to be insufficient in inducing slower tumor growth. An integration of three methods (16S rRNA gene sequencing, metagenomic shotgun sequencing, and quantitative polymerase chain reaction (qPCR) for selected bacteria) revealed ten species that were differentially enriched in $\mathrm{R}$ versus NR groups. Eight bacterial species abundant in the R group, included Enterococcus faecium, Collinsella aerofaciens, Bifidobacterium adolescentis, Klebsiella pneumoniae, Veillonella parvula, Parabacteroides merdae, Lactobacillus sp., and Bifidobacterium longum. Ruminococcus obeum and Roseburia intestinalis were abundant in the NR group. This again complies with the study by Sivan and colleagues, who reported Bifidobacteriaceae family members to be associated with better immune-mediated tumor control and positive efficacy of the anti-PD-L1 therapy in mice. Monocolonization with the $\mathrm{R}$ group-enriched species resulted in decreased frequency of the colonic regulatory $\mathrm{T}$ cells, increased frequency of Batf3-lineage DCs, and enhanced Th1 response. Thus, the data suggests that the composition of the commensal microbiota plays a significant role in determining the therapeutic efficacy of anti-PD-1 monoclonal antibody and, hence, can be used as a biomarker for clinical response.

Daillere et al. announced two bacterial species, E. hirae and Barnesiella intestinihominis, that play an important role in determining the antitumoral efficacy of cyclophosphamide (CTX), an anti-cancer immunomodulatory agent (Daillère et al. 2016). E. hirae, a gram-positive bacteria residing in the small intestine, was found to selectively restore the CTX-mediated antitumor effects in ATB-treated mice and improved the cognate anticancer immune response. E. hirae specifically induced the systemic effector pathogenic Th17 (pTh17) cell responses related to MHC class I-restricted cytotoxic T cells (CTL) and significantly decreased the level of Foxp $3^{+} \mathrm{CD} 25^{+} \mathrm{CD} 4^{+}$TILs resulting in increased intratumoral CTL/T regulatory (Treg) cell ratio. The gramnegative $B$. intestinihominis, residing in the colon, enhanced the systemic polyfunctional $\mathrm{CD} 4^{+} \mathrm{Tc} 1$ and $\mathrm{CD} 8^{+} \mathrm{Th} 1$ cell responses by augmenting the proportions of intratumoral IFN-y-producing ydTILs while decreasing IL-17-producing ydTILs. In the spleen, E. hirae and B. intestinihominis, representing oncomicrobiotics (OMBs), facilitated the accumulation of effector $\mathrm{CD} 8^{+} \mathrm{T}$ cells by inverting the $\mathrm{CD} 4 / \mathrm{CD} 8 \mathrm{~T}$ cell ratio. Repetitive gavages of either OMB ameliorated the CTX activity against MCA-205 fibro-sarcoma. Gavages with B. intestinihominis were also efficacious against Ret melanoma. Daillere and colleagues found that IFN- $y$ neutralization with unaltered IL-17 or $\mathrm{CD} 8^{+} \mathrm{T}$ cell depletion critically debilitated the anticancer efficacy of the two commensals against MCA-205. Therefore, these OMBs exhibit the effect of an adjuvant on the tumoral immune responses, enhancing the efficacy of CTX. The authors also discovered that the immune-dependent anti-sarcoma effects of CTX were considerably enhanced in nucleotide oligomerization domain 2-deleted $\left(\mathrm{Nod}^{-/-}\right)$mice. CTX-treated sarcoma growing in Nod $2^{-/-}$mice showed lower number of Treg cells and higher number of IFN- $\gamma$-producing ydTILs compared to sarcoma growing in a wild type environment. Hence, NOD2 receptors were suggested to function as "gut immune checkpoints" that limited the immunogenicity of E. hirae and B. intestinihominis. Therefore, NOD2 hinders the accumulation of the two OMBS and restricts their proapoptotic effects on the epithelial cells. The study also concluded the immune response of memory Th1 toward E. hirae or B. intestinihominis to govern the progression-free survival in cancer patients.

Gopalakrishnan et al. investigated the oral and gut microbiome of melanoma patients who received anti-PD-1 immunotherapy based on a previous research finding that reported gut microbiome to modulate the tumor response to ICI treatment (Gopalakrishnan et al. 2018). They analyzed the patient's fecal microbiome samples and assessed the relative abundance of bacteria in the R and NR groups. The bacterial population was significantly different between the two groups. The $\mathrm{R}$ group exhibited substantially higher alpha diversity and higher frequency of bacteria belonging to the Ruminococcaceae family $(p<0.01)$ than the NR group. This favorable gut microbiome exhibited improved antitumor immunity in $\mathrm{R}$ patients and fecal transplanted germ-free mice. High abundance of Faecalibacterium sp. in the $\mathrm{R}$ group heightened the antigen presentation and $\mathrm{T}$ cell function in the tumor microenvironment, thereby, enhancing the antitumor immune responses. On the other hand, high relative abundance of Bacteroidales was associated with decreased antitumor immune responses owing to the diminished antigen presentation capacity. While increased activation of $\mathrm{CD}^{+}$T cells was observed in R-FMT mice, the spleen of NR-FMT mice exhibited increased levels of regulatory $\mathrm{CD} 4^{+} \mathrm{FoxP}^{+} \mathrm{T}$ cells and $\mathrm{CD} 4 \mathrm{IL}-17 \mathrm{~T}$ cells. This suggests that FMT from NR resulted in impaired host immune responses. The study emphasized on the therapeutic potential of regulating microbial composition in patients 
undergoing ICI therapy. While Gopalakrishnan et al., Routy et al., and Matson et al. studied the link between gut microbiome and efficacy of anti-PD-1 mAb in solid cancers (Gopalakrishnan et al. 2018; Matson et al. 2018; Routy et al. 2018), Matson et al.'s study was an exception owing to the different method used to identify the R and NR patients (Matson et al. 2018).

In a similar study, Tanoue et al. studied 11 bacterial strains that were positively associated with the frequency of IFN- $y$-producing CD8 T cells. These bacterial strains, isolated from healthy human donor feces, were mixed inoculated into germ-free mice (GF+11-mix mice) (Tanoue et al. 2019). A substantial increase in IFN- $\gamma^{+}$CD8 T cells was found in diverse genetic backgrounds, with relative enrichment for the $\mathrm{T}$ cell receptor (TCR) $\mathrm{V} \beta 6^{+}$and $\mathrm{V} \beta 8^{+}$subsets. GF+11-mix mice also exhibited induction of CD4 T cells, increasing the frequencies of colonic Th17 and Th1 cells as well. Among these 11 strains, 7 were Bacteroidales, which when mixed and inoculated into germ-free mice (7-mix) were incapable of inducing IFN- $y+\mathrm{CD} 8 \mathrm{~T}$ cells. The mix of the other 4 strains, which were non-Bacteroidales, although exhibited a better induction capacity, but still was not sufficient to yield the complete inductive effect of the 11-mix. Hence, it was suggested that the 11 strains, including the 7 Bacteroidales as supporting strains and the 4 nonBacteroidales as effector elements, formed a consortium. The upregulation of chemokines $\mathrm{CXCl} 9, \mathrm{CXCl10}$, and IFNinducible genes in the colonic epithelial cells by the colonization of the 11-mix suggested that the recruitment and accumulation of IFN- $y+\mathrm{CD} 8 \mathrm{~T}$ cells can be promoted by active colonization near epithelial cells, establishing an IFN$\gamma$-mediated feed-forward loop. Subcutaneous engraftment of GF+11-mix mice with MC38 adenocarcinoma cells showed significantly improved anti-PD-1 treatment efficacy, compared to that in germ-free mice and in mice with strains that do not induce IFN $-\gamma^{+}$CD8 T cells. This therapeutic effect was a result of increased IFN- $\gamma^{+}$CD8 TILs, as the depletion of $\mathrm{CD} 8 \mathrm{~T}$ cell by antibody notably reduced the efficacy. Interestingly, the colonization of 11-mix even suppressed the tumor growth without anti-PD-1 treatment. Altogether, this data demonstrates that the 11 strains with non-inflammatory immunomodulatory activity enhance both ICI-mediated and spontaneous anti-tumor immunity in a CD8 T cell-dependent manner. Evaluating the abundance of the 11 strains using metagenomic datasets, it was observed that majority of the strains were rare and low-abundance components of the human microbiota. The low-abundance trend was also established in both $\mathrm{R}$ and NR with melanoma receiving ICI treatment in the studies by Gopalakrishnan et al. (2018), Routy et al. (2018) and Matson et al. (2018).

Likewise, Pushalkar et al. established a specific gut microbiome that is linked to the progressive pancreatic oncogenesis in mice (Pushalkar et al. 2018). Analyzing the gut microbiome using 16S rRNA fluorescence in-situ hybridization (FISH) and qPCR, it was observed that both the mouse and human pancreatic ductal adenocarcinoma (PDA) exhibit significantly greater bacterial abundance compared to the normal pancreas. The most abundant of the 13 phyla that were detected in the human PDA were Proteobacteria, Bacteroidetes, and Firmicutes. The PDA-associated microbiome differentially activated selective Toll-like receptors (TLR) in order to create a tolerogenic immune program. The microbiome was observed to render suppressive effects on the T-cell activation when polarized on macrophages. However, in the absence of TLR signaling, this effect was attenuated. Bifidobacterium pseudolongum, differentially abundant in both gut and tumor, was also reported to accelerate oncogenesis in a TLR-dependent manner. Further, the macrophages polarized by cell-free extracts of $B$. pseudolongum upregulated the tolerogenic cytokine IL-10. Ablation of the gut bacteria by oral administration of antibiotics resulted in an increased activation of Th1 differentiation of both of $\mathrm{CD} 4^{+}$ and $\mathrm{CD} 8^{+} \mathrm{T}$ cells and decreased immune-suppressive cells. Thus, the ablation was associated with decrease in immunesuppressive $\mathrm{CD}_{206^{+}} \mathrm{M} 2$-like tumor-associated macrophages (TAM), an adjuvant to increase M1-like TAMs, which express MHC II, CD86, tumor necrosis factor alpha (TNF$\alpha$ ), IL-12, and IL-6. High expression of T-BET, TNF- $\alpha$, and IFN-y indicated an enhanced cytotoxic phenotype of $\mathrm{CD}^{+}$ $\mathrm{T}$ cells. The authors, thus, suggested bacterial ablation to create a synergistic efficacy with PD-1-directed therapies. Bacterial transfer from PDA-bearing hosts reversed the protective effect of microbial ablation against PDA. However, the reversion was not evident in a transfer from control-host; thus, emphasizing on the pro-tumorigenic effect of PDAassociated microbiome.

\section{Metabolites as a mechanism of modulating host cytokines and anticancer therapeutics}

While the specific functions and molecular mechanism of the gut microbiome are yet largely unidentified, Lee et al. explored a few of the strain-specific mechanisms of how the microbiome affects cancer development and the efficacy of anti-cancer therapy (Lee et al. 2021). Lee et al. found that the increase in anticancer immunity due to B. bifidum strainspecific enhancement of anticancer immunity is a result of IFN- $\gamma$ signaling. In addition, the $B$. bifidum-induced IFN- $\gamma$ production was enhanced by the increase in serum metabolites and peptidoglycan biosynthesis.

Lee et al. collected intestinal tissues from tumor mice treated with anti-PD-1 plus synergistic $B$. bifidum strains (B. bif_K57) or non-synergistic strains (B. bif_B06). By performing RNA sequencing intestinal tissues, they were able to observe a notably higher number of genes associated with Gene Ontology terms "lymphocyte activation", 
"interferon-gamma production" and "positive regulation of interferon-gamma production" in mice treated with antiPD-1 with B. bif_K57 compared to those treated with B. bif_B06. Serum metabolic profiling showed that the concentrations of L-tryptophan, uric acid, and $\mathrm{N}$-acetyl zonisamide were higher in mice treated with anti-PD-1 plus B. bif_K57 compared to mice treated with anti-PD-1 plus B. bif_B06. It was also found that in vitro, the addition of L-tryptophan increased IFN- $\gamma$ production from activated CD8+ T cells. Mice treated with anti-PD-1 plus B. bif_B57 exhibited the lowest serum lipid levels, followed by mice treated with antiPD-1 plus B. bif_B06 and then anti-PD-1. This, in line with a previous study that demonstrated that lipid challenge leads to the decrease in T cell secretion of IFN- $\gamma$ (Guerrero-Ros et al. 2020), shows that lowering the lipid level is an example of a mechanism by which the microbiome modulates cytokines.

Lee et al. also discovered that the peptidoglycan levels were significantly higher in B. bif_K57 compared with B. bif_B06. MurE and GlmM, which are the genes associated with the peptidoglycan biosynthetic process, were expressed more strongly in anti-PD-1 plus B. bif_K57-treated mice stools compared to anti-PD-1 plus B. bif_B06-treated mice. In patients with NSCLC, the stool samples of patients containing the B. bifidum exhibited enriched peptidoglycan biosynthesis pathway, compared to bifidum-free patients, demonstrating the effect of peptidoglycans on immune modulation.

\section{Conclusion}

The findings of the different studies discussed in this review highlighted a strong correlation between the gut microbiota and immune system. The significant role of the gut microbiome in regulating the antitumor activity of ICIs was also elucidated. Notably, species belonging to the genera Akkermansia (Routy et al. 2018; Xu et al. 2020), Enterococcus (Daillère et al. 2016; Matson et al. 2018; Routy et al. 2018), Bifidobacterium (Matson et al. 2018; Pushalkar et al. 2018; Sivan et al. 2015) and Ruminococcaceae (Gopalakrishnan et al. 2018; Tanoue et al. 2019) were found to improve the immune responses and increase the efficacy of PD-1 blockade therapy. Interestingly, the genus Bacteroides has been found to induce biphasic effects. While some strains of Bacteroides increase the response to CTLA-4 blockade (Vétizou et al. 2015), other strains of this genus are associated with negative therapeutic efficacy of PD-1 antibody immunotherapy (Xu et al. 2020). Gut microbial changes induce changes in the expression of cytokines and immune cells, resulting in differential therapeutic responses. Particular cytokines, IFN-y (Daillère et al. 2016; Garris et al. 2018; Matson et al. 2018; Routy et al. 2018; Tanoue et al.
2019; Xu et al. 2020), IL-12 (Garris et al. 2018; Routy et al. 2018; Vétizou et al. 2015), and IL-17 (Daillère et al. 2016; Gopalakrishnan et al. 2018), have been reported as the key cytokines that are used by certain bacterial strains to affect the immunomodulation. Additionally, immune cells such as the $\mathrm{CD}^{+}$T cells (Matson et al. 2018; Pushalkar et al. 2018; Sivan et al. 2015; Tanoue et al. 2019) and CD4 ${ }^{+}$T cells (Routy et al. 2018; Gopalakrishnan et al. 2018; Daillère et al. 2016; Pushalkar et al. 2018) have also been identified to be responsible for the responsiveness to ICP blockade therapy. The mechanism behind the strain-specific enhancement of anticancer immunomodulation was studied by Lee et al., in which they found that the IFN- $\gamma$ signaling was the key to the B. bifidum strain-specific anticancer immunity (Lee et al. 2021). More importantly, they discovered that the increase in serum L-tryptophan, along with other serum metabolites, and peptidoglycan biosynthesis augmented the B. bifiduminduced IFN- $\gamma$ production.

While some cytokines reveal the possible mechanism by which specific bacterial strains confer increased efficacy of ICI therapy, future studies are warranted to elucidate the detailed molecular mechanism of the mutual interaction. In addition, a more comprehensive analysis of the microbial composition associated with anticancer response will be necessary to better understand the link between the microbiome and their immunomodulatory effects. The isolated strains carry significant biotherapeutic potentials: they can be utilized as biomarkers to predict the efficacy of ICI and can also be applied to treat cancer and other related diseases.

Bacterial components are well-known pathogen-associated molecular patterns (PAMPs) that induce the innate immune system via the TLRs. However, antitumor immunity is usually exerted by the adaptive immune system, such as CD8 cytotoxic T cells specific for cancer peptides. Thus, it is important to clarify how the PAMPs activate cancer-specific adaptive immunity. Furthermore, while certain bacterial species enhance the efficacy of ICIs, some repress its efficacy, implying that the antitumor activity of the bacteria may not stem from the simple activation of the innate immune system by common PAMPs, such as lipopolysaccharide (LPS). More detailed understanding on how gut microbiota controls the systemic immune system is essential for better understanding and development of efficient therapeutics against cancers that lack efficient treatment strategies.

Acknowledgements This research was supported by the Bio \& Medical Technology Development Program of the National Research Foundation (NRF), funded by the Ministry of Science \& ICT (Grant Number: NRF-2017M3A9F3046536), a GIST Research Institute (GRI) grant, funded by the GIST in 2020. This research was supported by grants from National Cancer Centre, Korea (NCC-1911267).

Author contributions EK and HA wrote the manuscript. HP revised the manuscript. 


\section{Declarations}

Conflict of interest The authors declare that they have no conflict of interest.

Open Access This article is licensed under a Creative Commons Attribution 4.0 International License, which permits use, sharing, adaptation, distribution and reproduction in any medium or format, as long as you give appropriate credit to the original author(s) and the source, provide a link to the Creative Commons licence, and indicate if changes were made. The images or other third party material in this article are included in the article's Creative Commons licence, unless indicated otherwise in a credit line to the material. If material is not included in the article's Creative Commons licence and your intended use is not permitted by statutory regulation or exceeds the permitted use, you will need to obtain permission directly from the copyright holder. To view a copy of this licence, visit http://creativecommons.org/licenses/by/4.0/.

\section{References}

Daillère R, Vétizou M, Waldschmitt N, Yamazaki T, Isnard C, PoirierColame V, Duong CP, Flament C, Lepage P, Roberti MP (2016) Enterococcus hirae and Barnesiella intestinihominis facilitate cyclophosphamide-induced therapeutic immunomodulatory effects. Immunity 45:931-943

Darvin P, Toor SM, Nair VS, Elkord E (2018) Immune checkpoint inhibitors: recent progress and potential biomarkers. Exp Mol Med 50:1-11

Eisenhauer EA, Therasse P, Bogaerts J, Schwartz LH, Sargent D, Ford R, Dancey J, Arbuck S, Gwyther S, Mooney M (2009) New response evaluation criteria in solid tumours: revised RECIST guideline (version 1.1). Eur J Cancer 45:228-247

Garris CS, Arlauckas SP, Kohler RH, Trefny MP, Garren S, Piot C, Engblom C, Pfirschke C, Siwicki M, Gungabeesoon J (2018) Successful anti-PD-1 cancer immunotherapy requires $\mathrm{T}$ cell-dendritic cell crosstalk involving the cytokines IFN- $\gamma$ and IL-12. Immunity 49(1148-1161):e1147

Gopalakrishnan V, Spencer CN, Nezi L, Reuben A, Andrews M, Karpinets T, Prieto P, Vicente D, Hoffman K, Wei S (2018) Gut microbiome modulates response to anti-PD-1 immunotherapy in melanoma patients. Science 359:97-103

Guerrero-Ros I, Clement CC, Reynolds CA, Patel B, Santambrogio L, Cuervo AM, Macian F (2020) The negative effect of lipid challenge on autophagy inhibits $\mathrm{T}$ cell responses. Autophagy $16(2): 223-238$

He X, Xu C (2020) Immune checkpoint signaling and cancer immunotherapy. Cell Res 30:660-669
Lee SH, Cho SY, Yoon Y, Park C, Sohn J, Jeong JJ, Park H (2021) Bifidobacterium bifidum strains synergize with immune checkpoint inhibitors to reduce tumour burden in mice. Nat Microbiol 6(3):277-288

Matson V, Fessler J, Bao R, Chongsuwat T, Zha Y, Alegre M-L, Luke JJ, Gajewski TF (2018) The commensal microbiome is associated with anti-PD-1 efficacy in metastatic melanoma patients. Science 359:104-108

Pardoll D (2015) Cancer and the immune system: basic concepts and targets for intervention. Semin Oncol 42:523-538

Pushalkar S, Hundeyin M, Daley D, Zambirinis CP, Kurz E, Mishra A, Mohan N, Aykut B, Usyk M, Torres LE (2018) The pancreatic cancer microbiome promotes oncogenesis by induction of innate and adaptive immune suppression. Cancer Discov 8:403-416

Routy B, Le Chatelier E, Derosa L, Duong CP, Alou MT, Daillère R, Fluckiger A, Messaoudene M, Rauber C, Roberti MP (2018) Gut microbiome influences efficacy of PD-1-based immunotherapy against epithelial tumors. Science 359:91-97

Sivan A, Corrales L, Hubert N, Williams JB, Aquino-Michaels K, Earley ZM, Benyamin FW, Lei YM, Jabri B, Alegre M-L (2015) Commensal Bifidobacterium promotes antitumor immunity and facilitates anti-PD-L1 efficacy. Science 350:1084-1089

Tanoue T, Morita S, Plichta DR, Skelly AN, Suda W, Sugiura Y, Narushima S, Vlamakis H, Motoo I, Sugita K (2019) A defined commensal consortium elicits CD8 T cells and anti-cancer immunity. Nature 565:600-605

Vétizou M, Pitt JM, Daillère R, Lepage P, Waldschmitt N, Flament C, Rusakiewicz S, Routy B, Roberti MP, Duong CP (2015) Anticancer immunotherapy by CTLA-4 blockade relies on the gut microbiota. Science 350:1079-1084

Waldmann TA (2003) Immunotherapy: past, present and future. Nat Med 9:269-277

Wei SC, Duffy CR, Allison JP (2018) Fundamental mechanisms of immune checkpoint blockade therapy. Cancer Discov 8:1069-1086

Xu X, Lv J, Guo F, Li J, Jia Y, Jiang D, Wang N, Zhang C, Kong L, Liu Y (2020) Gut microbiome influences the efficacy of PD-1 antibody immunotherapy on MSS-type colorectal cancer via metabolic pathway. Front Microbiol 11:814

Yi M, Jiao D, Xu H, Liu Q, Zhao W, Han X, Wu K (2018) Biomarkers for predicting efficacy of PD-1/PD-L1 inhibitors. Mol Cancer 17:1-14

Zhang J-M, An J (2007) Cytokines, inflammation and pain. Int Anesthesiol Clin 45:27

Publisher's Note Springer Nature remains neutral with regard to jurisdictional claims in published maps and institutional affiliations. 